\title{
Estimation of inhaled ultrafine particle surface area dose for urban environments
}

\author{
S. Clifford ${ }^{1} \quad$ M. Mazaheri ${ }^{2} \quad$ E. R. Jayaratne ${ }^{3}$ \\ $\begin{array}{lll}\text { M. A. Megat Mokhtar } & \text { F. Fuoco } & \text { G. Buonanno }\end{array}$ \\ L. Morawska ${ }^{7}$
}

(Received 18 December 2013; revised 8 October 2014)

\begin{abstract}
There is considerable scientific interest in personal exposure to ultrafine particles. Owing to their small size, these particles are able to penetrate deep into the lungs, where they may cause adverse respiratory, pulmonary and cardiovascular health effects. This article presents Bayesian hierarchical models for estimating and comparing inhaled particle surface area in the lung.
\end{abstract}

http://journal.austms.org.au/ojs/index.php/ANZIAMJ/article/view/7819 gives this article, (C) Austral. Mathematical Soc. 2014. Published November 4, 2014, as part of the Proceedings of the 11th Biennial Engineering Mathematics and Applications Conference. ISSN 1446-8735. (Print two pages per sheet of paper.) Copies of this article must not be made otherwise available on the internet; instead link directly to this URL for this article. 


\section{Contents}

1 Introduction

C438

2 Methodology

C439

2.1 Inhaled dose . . . . . . . . . . . . . . . . . . . C439

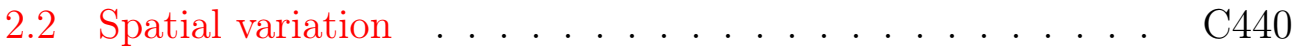

2.3 Microenvironment proportions . . . . . . . . . . . C441

3 Discussion

C443

References

C445

\section{Introduction}

Ultrafine particles (UFPs), particles less than $100 \mathrm{~nm}$ in diameter, account for the overwhelming majority of the number of outdoor airborne particles in Brisbane, Australia, the majority of which are generated by traffic [10]. While recent studies are inconclusive on the health effects of UFPs, the link between air quality and health is well known [5]. UFPs pose a unique risk, compared to coarser particles, as they can be inhaled deep into the lungs where they may be absorbed into the bloodstream [11].

High frequency measurements of ultrafine particle number concentration (PNC) with mobile sampling devices such as the Philips Nanotracer [8] estimate personal exposure to UFPs [3, 4, 9]. Such devices sample from a location on the person's body, rather than a fixed location in an environment where the person spends a large portion of time. The total daily personal dose is apportioned to the various microenvironments which are determined from an activity diary or from directly measured spatial information such as GPS coordinates.

This article describes a modelling methodology for estimating the inhaled 
particle surface area dose deposited within the lung. The model is based on size-specific particle deposition within the different regions of the lung, measurements of PNC in the breathing zone, and known and estimated parameters describing the particle size distribution.

\section{Methodology}

Bayesian regression models are developed to estimate inhaled doses in a number of microenvironments across a study region, the daily dose portion in each microenvironment, and the dose intensity relative to the time spent in each microenvironment. Here, all Bayesian models are estimated using R statistical software and Just Another Gibbs Sampler (JAGS) through the RJAGS package [12]. JAGS simulates the posterior distribution of the model parameters using Markov chain Monte Carlo (MCMC) techniques. The model code is provided in the supplementary material ${ }^{1}$.

\subsection{Inhaled dose}

The model for inhaled particle surface area dose in the alveolar (AL) and tracheo-bronchial (TB) regions of the lung extends a previous model [3] to incorporate the particle size distribution. The efficiency of particle deposition in the lung $\varphi$ varies with particle diameter $x_{p}$ in micrometres, and region of the lung [6],

$$
\begin{aligned}
& \varphi_{\text {AL }}\left(x_{p}\right)=\frac{3.52 \times 10^{-3}}{x_{p}}\left(e^{-0.234\left(\log x_{\mathfrak{p}}+3.40\right)^{2}}+63.9 e^{-0.819\left(\log x_{\mathfrak{p}}-1.61\right)^{2}}\right), \\
& \varphi_{\text {ТВ }}\left(x_{p}\right)=\frac{1.55 \times 10^{-2}}{x_{p}}\left(e^{-0.416\left(\log x_{\mathfrak{p}}+2.84\right)^{2}}+19.11 e^{-0.482\left(\log x_{\mathfrak{p}}-1.362\right)^{2}}\right) .
\end{aligned}
$$

${ }^{1}$ http://anziamj . austms.org. au/ojs/index.php/ANZIAMJ/article/ downloadSuppile/7819/24521 RJAGS model code. 
Personal air quality samplers do not report the full particle size distribution for a sample of air, but it is reconstructed by assuming a log-normal density $f\left[x_{p} ; \overline{x_{p}}(t), \sigma_{k}\right]$ with a location parameter based on the average particle diameter $\overline{x_{p}}(t)$ (measured by the Nanotracer) and some shape parameter $\sigma$. The particle size distribution shape parameter varies across the different microenvironments and is estimated based on prior studies with a Scanning Mobility Particle Sizer (SMPS) system [4]. An individual's inhalation rate $I_{k}$ also varies with microenvironment $k$, based on the type of activity engaged in at the time, as well as age. These inhalation rates are estimated from previous studies [4].

For individual $i$ in microenvironment $k$, the total inhaled surface area dose in the region of lung $r$ is

$$
d_{i k r}=\sum_{j=1}^{J} I_{k} \int_{T_{j k 1}}^{T_{j k 2}} \int_{0}^{\infty} N(t) f\left[x_{p} ; \overline{x_{p}}(t), \sigma_{k}\right] \varphi_{r}\left(x_{p}\right) \pi x_{p}^{2} d x_{p} d t,
$$

where $T_{j k 1}$ and $T_{j k 2}$ are, respectively, the start and end times of block $j$ of $J$ which the individual spends in microenvironment $k$, and $N(t)$ is the PNC as measured by the Nanotracer.

The model is implemented in $\mathrm{R}$ by looping over microenvironments within a loop for individuals (identified by a unique ID), selecting a block of contiguous time spent in a microenvironment, and calculating the double integral in equation (2). Integration over the range of particle diameters, from zero to infinity, is performed using adaptive quadrature in R's integrate function; time integration is performed using the trapezoid rule. Integration with an unbounded upper limit ensures that the entire mass of the log-normal density $f$ is included even though it is almost zero beyond $3000 \mathrm{~nm}$.

\subsection{Spatial variation}

To analyse the relationship between the inhaled doses within different study area subregions and microenvironments, Bayesian hierarchical linear models 
are developed. Bayesian hierarchical linear modelling casts the parameters as random variables described by a probability density. This is in contrast to classical ANOVA and linear regression models which assume that model parameters are unknown but fixed and rely on asymptotic properties of estimators. The incorporation of a priori beliefs about these parameters, whether assumed to be informative or not, is done by specifying a prior distribution for the parameters. These prior distributions are updated by the data to obtain a distribution representing a posteriori beliefs.

To estimate how microenvironment doses varies between subregions (or cohorts), a model with exchangeable random effect means is formulated as

$$
\begin{aligned}
\left(\mathrm{d}_{\mathrm{ik}} \mid z_{\mathrm{i}}=\mathrm{j}\right) & \sim \log \mathcal{N}\left(\beta_{\mathrm{jk}}, \tau_{\mathrm{d}}\right), & \beta_{\mathrm{jk}} & \sim \mathcal{N}\left(\alpha_{\mathrm{k}}, \tau_{\mathrm{k}}\right), \\
\alpha_{\mathrm{k}} & \sim \mathcal{N}\left(0,10^{-6}\right), & \tau_{\mathrm{d}}, \tau_{\mathrm{k}} & \sim \Gamma(0.001,0.001),
\end{aligned}
$$

where $\beta_{j k}$ is a location parameter for dose in microenvironment $k$ within spatial subregion $z_{i}=\mathfrak{j}$, and $\alpha_{k}$ is the all-region location parameter for each microenvironment with a conjugate weakly informative prior. The normal distributions are parameterised in terms of a mean (location) and precision (shape; inverse of the variance) and weakly informative conjugate Gamma distributions are applied to the precision parameters. A comparison of the dose received in two different microenvironments (e.g., $k=1,2$ ) for any given spatial subregion is given by calculating the posterior difference in means $\gamma_{j}=\beta_{j 1}-\beta_{j 2}$. This model will allow comparisons to be made across subregions and across microenvironments similar to an ANOVA but without assuming independence of the levels of the grouping variables; individuals are assumed exchangeable within subregions and these subregions are also exchangeable within each microenvironment type.

\subsection{Microenvironment proportions}

To estimate the average proportion of each individual's total daily dose received in each of the microenvironments, a multinomial model is developed. 
The multinomial model ensures that credible intervals for all proportion parameters remain within the range $[0,1]$. The model assumes exchangeability across individuals and the parameters of interest are the multinomial proportions $p_{k}$ derived from the parameters of the multinomial distribution $\theta_{k}$. Each individual's dose in each microenvironment $d_{i k}$ is some proportion of their 24 hour total inhaled dose $n_{i}$,

$$
\begin{aligned}
y_{i k} & =\left\lfloor d_{i k} / n_{i} \times 1007, \quad n_{i}=\sum_{k=1}^{k} d_{i k}, \quad y_{i 1} \sim \operatorname{Bin}\left(p_{1}, n_{i}\right),\right. \\
\vec{\theta} & \sim \operatorname{Dirichlet}(\vec{\alpha}), \quad p_{1}=\theta_{1}, \quad \alpha_{k} \sim \Gamma(2,2), \\
y_{i k} & \sim \operatorname{Bin}\left(p_{k}, n_{i}-\sum_{m=1}^{k-1} d_{i m}\right), \quad p_{k}=\theta_{k}\left(k-\sum_{m=1}^{k-1} \theta_{m}\right)^{-1},
\end{aligned}
$$

for $k \neq 1$ in the last two equations. The multinomial distribution generalises the binomial distribution to more than two categories and is parameterised as a series of binomials. This parameterisation is required as JAGS is not able to fit a multinomial model with zero counts [1], such as when an individual does not spend any time within a particular microenvironment. The Dirichlet prior for the multinomial proportions $\vec{\theta}$ represents a belief about the relative proportions in each microenvironment in the absence of data. The Gamma prior for the Dirichlet parameters $\vec{\alpha}$ results in a weakly informative conjugate prior which assumes that the proportions are equal a priori.

This model is also applied to the proportion of time spent in each microenvironment. Each individual's dose intensity for each microenvironment is the ratio of daily dose portion to daily time portion:

$$
\rho_{i k}=\frac{d_{i k}}{\sum_{k=1}^{K} d_{i k}} / \frac{\sum_{j=1}^{J} T_{j k 2}-T_{j k 1}}{\sum_{k=1}^{K} \sum_{j=1}^{J} T_{j k 2}-T_{j k 1}},
$$

which is a dimensionless number greater than zero, and indicates which microenvironments pose the greatest risk of an adverse health outcome. A 
dose intensity greater than one indicates that the individual received a higher portion of their total daily dose in that microenvironment than the portion of the day that they spent there. Inference on average dose intensities for each microenvironment $\widehat{\rho}_{k}$ are obtained by fitting

$$
\rho_{i k}, \widehat{\rho}_{k} \sim \log \mathcal{N}\left(\beta_{k}, \tau_{k}\right), \quad \beta_{k} \sim \mathcal{N}\left(0,10^{-4}\right), \quad \tau_{k} \sim \Gamma(0.001,0.001),
$$

where the dose intensities $\rho$ are assumed to be independent across the microenvironments and are distributed log-normally with location parameter $\beta$ and shape parameter $\tau$.

\section{Discussion}

The Bayesian hierarchical linear models developed here allow analysis which is more flexible than classical ANOVA approaches. By including spatial variation with a structured random effect mean, which is assumed exchangeable rather than independent across spatial locations, small area estimates of mean inhaled doses are calculated which are related to each other and informed by partial pooling of the data through the hierarchical model.

The multinomial model with MCMC sampling provides credible intervals for the proportion parameters which are strictly between zero and one and do not rely on assumptions of asymptotic behaviour. These are desirable properties for intervals with uncertainty in parameters which may be close to zero and are based on a small number of observations.

The dose model presented here extended previous work on dose calculation [3] by including particle size distribution estimation based on shape parameters [4]. This modelling approach was used to calculate the inhaled surface area dose in the alveolar and tracheo-bronchial regions of the lung of children attending 25 primary schools in the Brisbane Metropolitan Area [9].

The biggest outstanding issue in this modelling is the estimation of the shape parameter for each microenvironment. Point estimates result in underesti- 
mating the variability of the inhaled doses. Replacing the point estimates with a prior distribution $\sigma_{j k}^{2} \sim p\left(\sigma_{j k}^{2}\right)$ gives a more physically realistic estimate. While portable size distribution analysers have existed for approximately 40 years [7], the technology is not yet at a point where a particle size distribution analyser can be worn comfortably without interfering with normal movement (the Grimm Model 1.109 aerosol spectrometer has 32 size channels in which it measures PNC and can be carried in a backpack but weighs $2.5 \mathrm{~kg}[2])$.

Because personal samplers such as the Philips Nanotracer do not currently measure the size distribution, the shape parameters must be derived from nearby stationary monitors such as an SMPS system. As such, the measurement of particle size distributions (and calculation of the geometric standard deviation of particles) makes dose calculations across a large spatial domain very difficult. For well mixed air in microenvironments where individuals spend much of their time, the models provided in this article can be used with stationary samplers such as condensation particle counters and SMPS which more fully characterise the particle number concentration and size distribution as they have larger ranges of measurement than personal samplers.

Acknowledgements This work was supported by the Australian Research Council, Queensland Department of Transport and Main Roads (DTMR) and Queensland Department of Education, Training and Employment (DETE) through Linkage Grant LP0990134. Sam Clifford is supported by a postdoctoral fellowship from the Centre for Air quality health Research and evaluation. The authors thank R. Fletcher (DTMR) and B. Robertson (DETE), G. Marks, P. Robinson, K. Mengersen, Z. Ristovski, G. Ayoko, C. He, G. Johnson, S. Low Choy, G. Williams, W. Ezz, F. Salimi, L. Crilley, N. Mishra, R. Laiman, L. Guo, C. Duchaine, H. Salonen, X. Ling, J. Davies, L. Leontjew Toms, A. Cortes, B. Toelle, A. Quinones, P. Kidd, E. Belousova, M. Falk, F. Fatokun, J. Mejia, D. Keogh, T. Salthammer, R. Appleby and C. Labbe. 


\section{References}

[1] I. Albert and J.-B. Denis. Dirichlet and multinomial distributions: properties and uses in JAGS. Unité Mathématiques et Informatique Appliquées, INRA, Technical Report 2012-5, 2012. http: //w3. jouy.inra.fr/unites/miaj/public/nosdoc/rap2012-5.pdf. C442

[2] A. V. Broich, L. E. Gerharz, and O. Klemm. Personal monitoring of exposure to particulate matter with a high temporal resolution. Environ. Sci. Pollut. R., 19:2959-2972, 2012. doi:10.1007/s11356-012-0806-3. C444

[3] G. Buonanno, G. Giovinco, L. Morawska, and L. Stabile. Tracheobronchial and alveolar dose of submicrometer particles for different population age groups in Italy. Atmos. Environ., 45(34):6216-6224, 2011. doi:10.1016/j.atmosenv.2011.07.066. C438, C439, C443

[4] G. Buonanno, L. Morawska, L. Stabile, L. Wang, and G. Giovinco. A comparison of submicrometer particle dose between Australian and Italian people. Environ. Pollut., 169:183-189, 2012. doi:10.1016/j.envpol.2012.03.002. C438, C440, C443

[5] HEI Review Panel on Ultrafine Particles. Understanding the Health Effects of Ambient Ultrafine Particles. HEI Perspectives 3, Technical Report, 2013. http://pubs . healtheffects.org/getfile.php?u=893. C438

[6] ICRP. Human Respiratory Tract Model for Radiological Protection. ICRP Publication 66. Ann. ICRP 24(1-3), 1994. http: //www . icrp.org/publication. asp?id=ICRP\%20Publication\%2066. C439 
[7] B. Y. H. Liu, K. T. Whitby, and D. Y. H. Pui. A portable electrical analyzer for size distribution measurement of submicron aerosols. J. Air Pollut. Control Assoc., 24(11):1067-1072, 1974. doi:10.1080/00022470.1974.10470016. C444

[8] J. Marra, M. Voetz, and H.-J. Kiesling. Monitor for detecting and assessing exposure to airborne nanoparticles. J. Nanopart. Res., 12(1):21-37, 2010. doi:10.1007/s11051-009-9695-x. C438

[9] M. Mazaheri, S. Clifford, R. Jayaratne, M. A. Megat Mokhtar, F. Fuoco, G. Buonanno, and L. Morawska. School children's personal exposure to ultrafine particles in the urban environment. Envir. Sci. Tech., 48(1):113-120, 2014. doi:10.1021/es403721w. C438, C443

[10] L. Morawska, S. Thomas, N. Bofinger, D. Wainwright, and D. Neale. Comprehensive characterization of aerosols in a subtropical urban atmosphere: particle size distribution and correlation with gaseous pollutants. Atmos. Environ., 32:2467-2478, 1998. doi:10.1016/S1352-2310(98)00023-5. C438

[11] G. Oberdörster. Pulmonary effects of inhaled ultrafine particles. Int. Arch. Occup. Environ. Health, 74(1):1-8, 2001. http://www.ncbi.nlm.nih.gov/pubmed/11196075. C438

[12] M. Plummer. RJAGS: Bayesian graphical models using MCMC, $\mathrm{R}$ package version 3-9. 2012.

http://cran.r-project.org/web/packages/rjags/index.html. C439

\section{Author addresses}

1. S. Clifford, Science and Engineering Faculty, Queensland University of Technology, Brisbane Queensland 4000, Australia.

mailto:samuel.clifford@qut.edu.au 
2. M. Mazaheri, Science and Engineering Faculty, Queensland University of Technology, Brisbane Queensland 4000, Australia.

3. E. R. Jayaratne, Science and Engineering Faculty, Queensland University of Technology, Brisbane Queensland 4000, Australia.

4. M. A. Megat Mokhtar, Science and Engineering Faculty, Queensland University of Technology, Brisbane Queensland 4000, Australia.

5. F. Fuoco, Department of Civil and Mechanical Engineering, University of Cassino and Southern Lazio, via Di Biasio 43, 03043 Cassino, Italy

6. G. Buonanno, Department of Civil and Mechanical Engineering, University of Cassino and Southern Lazio, via Di Biasio 43, 03043 Cassino, Italy

7. L. Morawska, Science and Engineering Faculty, Queensland University of Technology, Brisbane Queensland 4000, Australia. 